\title{
IMPACTO DE LAS CLÁUSULAS AMBIENTALES DE LOS ACUERDOS COMERCIALES REGIONALES SOBRE LOS NIVELES DE CONTAMINACIÓN
}

\author{
Alan Fairlie Reinoso \\ Pontificia Universidad Católica del Perú \\ ORCID: 0000-0001-7584-2374 \\ Jessica Milagros Portocarrero Torres \\ Universidad San Martín de Porres \\ ORCID: 0000-0003-0541-6753 \\ Tania Karen Paredes Zegarra \\ Pontificia Universidad Católica del Perú \\ ORCID: 0000-0003-0190-9753
}

\begin{abstract}
Resumen: El cambio climático, y el impacto del modelo económico en el medio ambiente, obliga a los países de la región a establecer diferentes herramientas y mecanismos para enfrentar sus efectos negativos. Los acuerdos regionales comerciales tienen un rol fundamental a través de la incorporación de cláusulas o disposiciones ambientales, que estén orientadas a garantizar la calidad ambiental. Este artículo busca determinar el impacto de las cláusulas ambientales de los acuerdos comerciales regionales, sobre los indicadores de contaminación ambiental de los países exportadores socios, especialmente en la Alianza del Pacífico.
\end{abstract}

Palabras claves: Cláusulas ambientales, Acuerdos comerciales regionales, Alianza del Pacífico, Contaminación, América Latina. 


\title{
Impact of Environmental Clauses of Regional Trade Agreements in the Pollution Levels
}

\begin{abstract}
Climate change and the impact of the economic model in the environment, forces the countries of the region to establish different tools and mechanisms to face its negative effects. For that reason, the environmental provisions of Regional Trade Agreements play a crucial role in guaranteeing environmental quality. This article estimates the impact of the environmental clauses of the regional trade agreements on the environmental pollution indicators of the partner exporting countries, especially in the Pacific Alliance.
\end{abstract}

Keywords: Environmental clauses, Trade agreements, Pacific Alliance, Pollution, Latin America. 


\section{Alan Fairlie Reinoso}

Doctor en Gestión Estratégica por la Pontificia Universidad Católica del Perú. Magíster en Comercio Internacional y Desarrollo en el Instituto Torcuato Di Tella, así como también magíster en Política y Gestión Universitaria y Licenciado en Economía de la PUCP. Profesor principal del Departamento de Economía de la Pontificia Universidad Católica del Perú (PUCP) y director de la Maestría en Biocomercio y Desarrollo de esta casa de estudios.

Correo: afairli@pucp.edu.pe

\section{Jessica Milagros Portocarrero Torres}

Economista por la Universidad San Martín de Porres y Maestrante en Ciencias Contables y Financieras con mención en Tributación Fiscal y Empresarial de dicha casa de estudios. Se ha desempeñado como asesora del Parlamento Andino para la representación del Perú. Así como Especialista de la Oficina de Cooperación y Asuntos Internacionales del Ministerio de Educación del Perú.

Correo: jessica.portocarrero@gmail.com

\section{Tania Karen Paredes Zegarra}

Economista por la Pontificia Universidad Católica del Perú y Maestrante en Economía con mención en Desarrollo Económico de dicha casa de estudios. Se ha desempeñado como jefe de prácticas en la Pontificia Universidad Católica del Perú. Así como técnico del Parlamento Andino para la representación del Perú.

Correo: tania.paredes@pucp.edu.pe 


\section{Introducción}

La pandemia por el COVID-19 ha acentuado la necesidad de avanzar hacia un nuevo modelo económico y de desarrollo, para hacer frente a las diferentes crisis que enfrentamos, entre ellas la ambiental. Los diversos problemas ambientales y de cambio climático tienen origen en el modelo económico productivo actual; asimismo, las vulnerabilidades asimétricas ante el cambio climático pueden poner en riesgo el cumplimiento de los Objetivos de Desarrollo Sostenible (ODS).

Ante estos problemas, los Estados deben explorar diferentes alternativas para poder contrarrestar su avance. Es en ese contexto donde los Acuerdos Comerciales Regionales (ACR) tienen un rol fundamental para impulsar acciones a favor de la lucha contra el cambio climático, a través de la incorporación de cláusulas o disposiciones ambientales que estén orientadas a garantizar la calidad ambiental de los países que lo suscriben. Es decir que la flexibilización de algunas de las barreras comerciales no tenga un efecto negativo sobre el medio ambiente.

En ese sentido, este artículo busca determinar el impacto de las cláusulas ambientales presentes en los Acuerdos Comerciales Regionales, sobre los indicadores de contaminación ambiental de los países exportadores que conforman dichos tratados. Primero se hace una breve revisión del impacto del comercio en el medio ambiente, para luego estimar un modelo de gravedad aumentado, partiendo de la metodología empleada por Baghdadi, MartínezZarzoso y Zitouna (2013), Ghosh y Yamarik (2006) y MartínezZarzoso (2018), a fin de determinar si el comercio impacta en el incremento o reducción de la contaminación ambiental durante el periodo 2000-2019. Se procederá a realizar la estimación a nivel de América Latina, para posteriormente enfocarnos en la Alianza del Pacífico.

\section{Implicancias del cambio climático en la región}

América Latina es una de las regiones del mundo que se caracteriza por su gran biodiversidad, pues alberga el 50\% del total mundial, 
situación que coloca a los países latinoamericanos en una posición más frágil y vulnerable al cambio climático (Grazzi \& Sasso 2020). Asimismo, la región latinoamericana presenta una gran asimetría entre el nivel de emisiones de gases de efecto invernadero y la vulnerabilidad; puesto que a pesar de solo ser responsable del 8.3\% de las emisiones a nivel mundial, son los más afectados ante el cambio climático.

La CEPAL (2020) estima que el costo económico del cambio climático en la región representará, para 2050, entre el 1.5\% y el 5\% del Producto Bruto Interno (PBI) regional actual. En ese sentido, resulta fundamental avanzar en los esfuerzos de adaptación, teniendo en cuenta las vulnerabilidades que presenta la región. Estudios como el realizado por la UNCTAD (2021b), sostienen que los costos asociados a la adaptación al cambio climático en los países en desarrollo oscilarán entre 140 y 300 mil millones de dólares anuales en 2030, y aunque los países desarrollados se comprometieron a movilizar conjuntamente 100 mil millones de dólares al año en financiamiento climático para abordar las necesidades de mitigación y adaptación (de los cuales solo 50 mil millones de dólares anuales estarían disponibles para la adaptación), estaríamos frente a una «brecha de adaptación», que deberá ser cubierta por los gobiernos nacionales y el sector privado en los países en desarrollo. Por lo cual, es importante que los países de la región exploren diferentes alternativas que les permitan disminuir los niveles de contaminación y otros factores asociados al cambio climático.

De otro lado, como consecuencia del cambio climático, las actividades económicas y comerciales a nivel mundial tienden a reconfigurarse, lo cual, a su vez, genera nuevas pautas de comercio, en donde los mayores beneficios se acumularán en las regiones polares y las mayores pérdidas en las regiones tropicales y subtropicales, donde se encuentran la mayoría de los países en desarrollo del mundo.

Los países en desarrollo son los que pierden una mayor parte de su riqueza, pues los efectos negativos de los eventos climáticos recaen de manera desproporcionada sobre la población más vulnerable, incrementado de esta forma los niveles de desigualdad y pobreza. Lo cual, a su vez, implica una menor capacidad para hacer frente a los efectos económicos del cambio climático (Hoffmann 2021).

Al respecto, Hoffmann sostiene que:

Las políticas de desarrollo deben ser coherentes con los objetivos de estabilización del clima y con los objetivos de gestión de riesgos de desastres, y que las políticas para ralentizar el cambio climático y gestionar los riesgos de desastres deben diseñarse para disminuir la desigualdad (2020: 249). 
Nuestra región enfrenta grandes desafíos para hacer frente a la crisis climática existente, así como la emergencia sanitaria que estamos viviendo a causa del COVID-19. Es necesario migrar hacia un modelo de desarrollo más sostenible, limpio y resiliente, para lo cual se requieren políticas que nos permitan avanzar en la adaptación y mitigación del cambio climático.

\section{Política comercial y medio ambiente}

La Organización Mundial de Comercio (OMC) busca contribuir a la protección y preservación del medio ambiente mediante la apertura del comercio, sus normas y mecanismos de observancia. Y el Comité de Comercio y Medio Ambiente busca determinar el efecto que las políticas comerciales puedan tener sobre el medio ambiente, así como las políticas ambientales sobre el comercio.

Cronológicamente, se reconoció la relación entre el comercio y la protección del medio ambiente en 1970, impulsada principalmente por la preocupación a nivel internacional sobre el impacto del crecimiento económico en el desarrollo social y el medio ambiente. Esto originó que en 1972 se establezca la Conferencia de Estocolmo, como respuesta a estas interrogantes. Posteriormente, se creó el Grupo MACI (Grupo de las Medidas Ambientales y el Comercio Internacional) en el Acuerdo General sobre Aranceles Aduaneros y Comercio (GATT). Las políticas ambientales empezaron a tener una mayor relevancia en el comercio internacional en la Ronda Uruguay (1986 a 1994), así como la conferencia de Río de Janeiro de 1992. Posteriormente, en 1994, se firmó la Decisión Sobre Comercio y Medio Ambiente, la misma que establecía la creación del Comité de Comercio y Medio Ambiente (CCMA) (OMC 2021a).

En la Ronda de Doha de 2001 se plantean expresamente cuestiones ambientales. El objetivo primordial es potenciar el apoyo mutuo del comercio y el medio ambiente, además de examinar la coexistencia armoniosa entre las normas de la OMC y las obligaciones comerciales específicas enunciadas en diversos acuerdos negociados a nivel multilateral para proteger el medio ambiente, e incluye algunas tareas para el CCMA (OMC 2021b).

De otro lado, la OMC está poniendo en práctica los lineamientos establecidos en el Objetivo de Desarrollo Sostenible 17: «Revitalizar la Alianza Mundial para el Desarrollo Sostenible de la Agenda 2030», particularmente la

[...] meta 17.10 Promover un sistema de comercio multilateral universal, basado en normas, abierto, no discriminatorio $\mathrm{y}$ equitativo en el marco de la Organización Mundial del Comercio, incluso mediante la conclusión de las negociaciones en el marco del Programa de Doha para el Desarrollo. 
En ese sentido, la protección del medio ambiente es un objetivo central del sistema multilateral del comercio. Entre 2009 y 2018, los Miembros de la OMC reportaron a esta institución más de 11 mil medidas ambientales, en contraste con lo registrado en 1995, donde menos de 1 de cada 12 notificaciones de medidas comerciales tenía un componente medioambiental. En la actualidad, este ratio es de 1 de cada 6 (OMC 2020).

El impacto del comercio en el medio ambiente es complejo. Por un lado, el comercio sin regulación efectiva podría llevar a una mayor contaminación ambiental. Por otro lado, la OMC resalta que mayores niveles de comercio pueden, potencialmente, generar mayores avances tecnológicos y ganancias de eficiencia que, en última instancia, promueven un desarrollo sostenible. Una de las críticas más importantes a este último argumento es que el transporte internacional de mercancías también contamina. Sin embargo, se conoce que, fruto de acciones coordinadas entre organismos internacionales, ya existen propuestas para reducir el impacto ambiental de todos los modos de transporte a través de una combinación de uso de energía renovable, tecnologías innovadoras, entre otras medidas (OMC 2020).

De otro lado, cada vez más, los acuerdos comerciales incluyen en sus negociaciones temas que van más allá del ámbito comercial, incorporando capítulos o títulos sobre transferencias tecnológicas, inversiones y desarrollo sostenible (Baumann 2016). Este tipo de acuerdos son denominados «Acuerdos Comerciales Profundos», pues son acuerdos recíprocos entre países que cubren no solo el comercio, sino también políticas adicionales destinadas a profundizar la integración económica entre los socios comerciales, abordando aspectos como protección de los derechos de propiedad intelectual, innovación, transferencia de tecnología, inversiones, trabajo decente y medio ambiente, entre otros (Mattoo, Rocha \& Ruta 2020). Asimismo, este tipo de acuerdos son conocidos como acuerdos de nueva generación al «añadir a los aspectos comerciales la preocupación por el respeto de estándares adecuados de protección ambiental y social, mediante los que se busca asegurar la sostenibilidad del desarrollo» (Manero 2020: 616).

Así, muchos de los acuerdos comerciales suscritos por los países latinoamericanos han incluido cláusulas o capítulos ambientales, a fin de aminorar los impactos que las actividades económicas propias del acuerdo comercial puedan ocasionar al medio ambiente. Además, se busca que los países no flexibilicen sus normativas ambientales para incentivar la atracción de inversiones o del comercio, aunque existe un amplio debate sobre este punto.

En el caso del Perú, los acuerdos comerciales suscritos con Estados Unidos y la Unión Europea contienen cláusulas vinculantes respecto a la protección y conservación del medio ambiente. Asimismo, el Acuerdo 
Transpacífico (TPP-11), y los acuerdos comerciales de Perú con Canadá y Australia, también incorporan capítulos o normativa asociados al tema ambiental (Salazar 2020).

Sin embargo, existe un debate acerca de la eficiencia de estas cláusulas. Desde la perspectiva de la sociedad civil, existe una preocupación en cuanto a la flexibilización de los estándares ambientales, promovidas con el fin de atraer inversiones extranjeras, que han debilitado los procedimientos que pretenden asegurar la protección ambiental.

Este aspecto se tendería a agudizar más en el actual contexto, puesto que, como parte del proceso de reactivación económica, los países vienen estableciendo normativas que no solo flexibilizan la protección ambiental, sino que impactan en los derechos colectivos al no establecer lineamientos que permitan o garanticen la implementación de la consulta previa y la participación ciudadana en los proyectos de inversión (CooperAcción 2020).

Por lo cual, la existencia de estos capítulos o cláusulas ambientales requieren de un adecuado seguimiento y monitoreo, a fin de garantizar su cumplimiento efectivo. En ese sentido, los espacios o mecanismos de consulta que algunos de estos acuerdos establecen son necesarios para propiciar la transparencia de la información ambiental en los acuerdos, especialmente en término de inversiones.

Pese a ello, existe evidencia de que la incorporación de cláusulas ambientales tiene un impacto indirecto en la calidad del medio ambiente. Ghosh y Yamarik (2006) encontraron que la suscripción de Tratados de Libre Comercio (TLC) reduce la contaminación, pero el efecto es indirecto: el aumento del comercio mejora el ingreso per cápita, y este, a su vez, la calidad del medio ambiente. Asimismo, de acuerdo con los hallazgos de Baghdadi, MartínezZarzoso y Zitouna (2013), quienes diferencian en su estudio aquellos acuerdos que cuentan con cláusulas ambientales de los que no, existe un efecto directo en la reducción de emisiones de $\mathrm{CO} 2$ en aquellos países que suscribieron los acuerdos comerciales que cuentan con este tipo de disposiciones ambientales.

También ha sido corroborado en el caso del Tratado Comercial suscrito entre la Unión Europea y Chile, ya que se establecieron normas ambientales más estrictas, y el comercio con la Unión Europea ha contribuido positivamente a la reducción de la intensidad de la contaminación de la producción de algunos sectores (Ergon Associates 2011).

Asimismo, Martínez-Zarzoso (2017) sostiene que los TLC con cláusulas medioambientales tienen un efecto positivo directo en la reducción de la degradación del medio ambiente, además de generar incentivos a los miembros para garantizar el cumplimiento de las regulaciones ambientales. Estima que los TLC con disposiciones ambientales o acuerdos paralelos reducen las emisiones de gases contaminantes. 
Por cada TLC adicional con contenido medioambiental la concentración media de PM2.5 (material particulado de 2.5 micras) ponderada por población disminuye en torno al $0.3 \%$, mientras que para SO2(Dióxido de azufre), NOx (dióxido de nitrógeno) y $\mathrm{CO} 2$ (dióxido de carbono) la disminución correspondiente de emisiones per cápita es de 2\%, 0.1\% y 0.6\%, respectivamente (MartínezZarzoso 2017: 172).

En ese sentido, y teniendo en cuenta la evidencia existente, los Acuerdos Comerciales Regionales tienen un potencial para contribuir a la conservación del medio ambiente, por lo cual es necesario que estas cláusulas se cumplan, a fin de promover un desarrollo sostenible y atender las externalidades ocasionadas, como el cambio climático, estrés ambiental y aumento de vulnerabilidades sistémicas (la crisis por el COVID-19, una de ellas).

En la siguiente sección haremos una estimación econométrica para evaluar el impacto de los acuerdos comerciales regionales con cláusulas medioambientales en la disminución de la contaminación ambiental durante el periodo 2000-2019. Se procederá a realizar la estimación a nivel de América Latina, para posteriormente enfocarnos en la Alianza del Pacífico.

\section{Evidencia empírica}

\subsection{Especificaciones del modelo}

La metodología para analizar el efecto de las cláusulas ambientales, presentes en los Acuerdos Comerciales Regionales (ACR) y en los indicadores de contaminación ambiental de los países exportadores que conforman dicho tratado, se basará en los trabajos empíricos realizados por Baghdadi, MartínezZarzoso y Zitouna (2013), Ghosh y Yamarik (2006) y Martínez-Zarzoso (2018). En estos se estima una ecuación de gravedad que permite identificar propiamente el efecto del comercio, para luego incluir este resultado en el análisis de los factores que determinan un incremento o reducción de la contaminación ambiental. En este sentido, se plantea la ecuación 1 sobre los determinantes de la contaminación ambiental en un país i en un periodo $t$.

$$
\begin{aligned}
& \ln \left(E_{i t}\right)=\delta_{i}+\gamma_{1} \ln \left(\text { Pop }_{i t}\right)+\gamma_{2} \ln \left(\text { GDPCap }_{i t}\right)+\gamma_{3} \ln \left(\text { GDPCap }_{i t}\right)^{2}+ \\
& \gamma_{4} \ln \left(\text { Open }_{i t}\right)+\gamma_{5} \sum_{j} w_{j t} * \text { rtaenv }_{i j t}+\gamma_{6} \sum_{j} w_{j t} * \text { rtanenv }_{i j t}+ \\
& \gamma_{7} \ln \left(\frac{\text { Area }}{\text { Pop }}\right)_{i t}+\theta_{t}+\mu_{i t}
\end{aligned}
$$


Donde $E_{i t}$ representa las emisiones per cápita de los contaminantes analizados del país $i$ en el periodo $t$ y estará expresado en logaritmos a fin de poder analizar la convergencia de las emisiones, y es la variable endógena del modelo. Por otro lado, $\delta_{i}$ es el término de constante específico del país $i$, Pop $p_{i t}$ será la medida del número de habitantes del país $i$ en el periodo $t$ y es un proxy para el efecto escala del modelo ${ }^{1}$. Por otro lado GDPCap ${ }_{i t}$ denota el PBI per cápita y su término cuadrado sirve para testear la hipótesis sobre las predicciones de que el incremento de la calidad ambiental eventualmente está relacionado con el ingreso. La variable explicativa $O p e n_{i t}$ se refiere al ratio de apertura del país que se mide como la suma de las exportaciones e importaciones dividido por el GDP (PBI) del país $i$ y este podría estar afectando positiva o negativamente la calidad ambiental.

Respecto a la variable explicativa de interés, rtanenv $v_{i j}$ denota el valor de 1 si el país $i$ y el país $j$ poseen un ACR con una cláusula ambiental vigente en el año $t$, y será 0 en otro caso. Por el contrario, $r$ tanenv $v_{i j}$ representa el valor de 1 si el país $i$ y el país $j$ poseen un ACR, pero esta no cuenta con una cláusula ambiental vigente en el año $t$, y será 0 en otro caso. La variable $w_{j t}$ es la ponderación dada a los diferentes Acuerdos Comerciales. No obstante, para este caso se asumirá que este será determinado por default por el modelo². Finalmente, $\frac{A r e a}{P o p}$ es el área percapita para el país $i$ en el periodo $t$ y $\theta_{t}$, denota una dummie que es proxy de la presencia de business cycles.

Por otro lado, dado que existe un efecto del comercio que es capturado por la variable $O$ pen $_{i t}$ y que podría estar generando un problema de endogeneidad por su correlación con variables como la población y el PBI en la ecuación 1, se realizará una estimación ex ante sobre esta variable de apertura a partir de un modelo de gravedad, como ha sido planteado también por Baghdadi, Martínez-Zarzoso y Zitouna (2013), Ghosh y Yamarik (2006) y Martínez-Zarzoso (2018). Por tal motivo, se plantea un modelo adicional para estimar en la ecuación (2) y (3), como se expresa a continuación:

$$
\ln \left(\text { Open }_{i t}\right)=\alpha_{1} \ln Y_{i t}+\alpha_{2} \ln Y_{j t}+\alpha_{3} \operatorname{lnPop}_{i t}+\alpha_{4} \ln P o p_{j t}+\tau_{i p}
$$

Donde

$$
\tau_{i p}=b_{1} \text { lndistance } i j+b_{2} \text { contig }+b_{3} \text { comlang_off }+b_{4} \text { colony }+b_{5} \text { comcol }
$$

En la ecuación 2, se tiene que el logaritmo natural del grado de apertura del país $i$ en el periodo $t$, depende tanto del PBI de los países $i$ y $j$ que conforman el flujo comercial en el periodo $t$, así como también de sus poblaciones respectivas en

El efecto escala indica si es que el aumento de la actividad económica por el comerciogenera un incremento en la contaminación y, por tanto, mayores daños ambientales.

2 Este supuesto se realiza en base a un análisis previo que ha sido realizado por Martínez-Zarzoso (2018). 
el mismo periodo. Por otro lado, existen otras variables explicativas referidas a características geográficas, como la distancia entre los países y la contigüidad de estos mediante fronteras, así como también aspectos culturales como una lengua común, la relación colonial entre países y la relación de colonización por el mismo país, que también influirán sobre la variable dependiente de esta primera etapa y que se especifica dentro de la ecuación (3).

Posteriormente, determinada la predicción sobre la variable de apertura, el logaritmo de la variable de apertura, ln ( open $\widehat{p e}_{i t}$ ), se procederá a incluir esta en la ecuación principal 1. Por lo que, finalmente, el modelo a estimar en la segunda etapa será el siguiente:

$$
\begin{aligned}
& \ln \left(E_{i t}\right)=\delta_{i}+\gamma_{1} \ln \left(\text { Pop }_{i t}\right)+\gamma_{2} \ln \left(\text { GDPcap }_{i t}\right)+\gamma_{3} \ln \left(\text { GDPcap }_{i t}\right)^{2}+ \\
& \gamma_{4} \ln \left(\text { Open }_{i t}\right)+\gamma_{5} \sum_{j} w_{j t} * \text { rtaenv }_{i j t}+\gamma_{6} \sum_{j} w_{j t} * \text { rtanenv }_{i j t}+ \\
& +\gamma_{7} \ln \left(\frac{\text { Area }}{\text { Pop }}\right)_{i t} \theta_{t}+\mu_{i t}
\end{aligned}
$$

La estimación del modelo principal que ha incorporado el valor predicho de la apertura y que será estimada para el análisis sobre las cláusulas ambientales, está expresada en la ecuación. La estimación se realizará a partir de la metodología de Datos Panel con Efectos Fijos intragrupo, que permitirá obtener la estimación del coeficiente en base a la relación del grupo sobre su media. Se opta por utilizar esta estrategia, dado que este método permitirá evitar cualquier problema de autocorrelación entre el término de error, respecto a los regresores. Por otro lado, para el caso de la ecuación de gravedad, se utilizará una estimación de efectos aleatorios.

\subsection{Base de datos y variables}

Los datos que se utilizarán para realizar el estudio provienen de las series del Banco Mundial (2021), la OECD (2021) y la UNCTAD (2021a). La base de datos contiene información para el periodo 2000-2019 para 19 países exportadores de América Latina y el Caribe, y para 52 países importadores de América Latina

Tabla 1. Países considerados en el análisis

\begin{tabular}{c|c}
\hline Países exportadores & Países importadores \\
\hline & Argentina, Australia, Austria, Bélgica, Belice, Bolivia, Brasil, \\
Argentina, Belice, Bolivia, Chile, & Canadá, Chile, China, Colombia, Costa Rica, Dinamarca, \\
Colombia, Costa Rica, Ecuador, & Ecuador, El Salvador, Estonia, Finlandia, Francia, Alemania, \\
El Salvador, Guatemala, Guyana, & Grecia, Guatemala, Honduras, Hungría, Islandia, Irlanda, \\
Honduras, México, Nicaragua, & Israel, Italia, Japón, Letonia, Luxemburgo, México, Países \\
Panamá, Paraguay, Perú, Surinam, & Bajos, Nueva Zelanda, Nicaragua, Noruega, Panamá, \\
Uruguay, Venezuela & Paraguay, Perú, Polonia, Corea, Rusia, Eslovaquia, Sudáfrica, \\
& España, Surinam, Suiza, Suecia, Turquía, Estados Unidos, \\
& Reino Unido, Uruguay y Venezuela \\
\hline
\end{tabular}


y el Caribe, Europa, Asia y Oceanía. Por otro lado, la frecuencia de los datos es anual, los cuales se especifican en la tabla 1.

Para el análisis de comercio norte-sur y sur-sur, la clasificación de países importadores según la estructura de comercio se toma de Naciones Unidas (2020).

La variable endógena es ellogaritmo del valor del contaminante ambiental, que para el análisis hará referencia a cuatro indicadores: i) dióxido de carbono, ii) óxido de nitrógeno, iii) dióxido de azufre y iv) material particulado de 2.5 micras. Estos valores se expresan en logaritmos, dado que lo que se quiere evaluar es la tasa de cambio en el tiempo sujeto a las variables explicativas del modelo. La información sobre el dióxido de carbono y óxido de nitrógeno se obtendrá de las series del Banco Mundial, mientras que la información del dióxido de azufre y material particulado de 2.5 micras ha sido obtenida de la base de datos de la OECD. La elección de las variables de contaminación ambiental estuvo sujeta a la disponibilidad de información sobre estos a nivel de país en el tiempo.

Por otro lado, la variable explicativa de interés es si los países poseen un Acuerdo Comercial Regional (ACR) con una cláusula que incluya una reglamentación ambiental. LA OMC define que la reglamentación ambiental consistirá en el desarrollo de estándares ambientales; aplicación de las leyes ambientales nacionales; establecimiento de sanciones por violación de leyes ambientales; publicaciones de leyes y reglamentos. El que un acuerdo tenga una cláusula de reglamentación ambiental se expresará como una variable dicotómica que tomará el valor de 1 cuando en el periodo de análisis t el par de países que comercian poseen una cláusula de reglamentación en el ACR y 0 en caso contrario (no cuenten con algún acuerdo). Complementariamente, también se construirá una variable que denote si el par de países posee un ACR, pero este no incluya una cláusula de cooperación. Esta información será obtenida del Banco Mundial y del documento de Martínez-Zarzoso (2018).

El detalle sobre los ACR que se ha considerado en el análisis se especifica a continuación (tabla 2).

Adicionalmente, en el caso de las variables de control, se incluye información del PBI del país exportador e importador, que estará expresado en millones de dólares a precios constantes de 2010; y la población del país exportador e importador, que estará expresado en millones de personas. La información sobre PBI y población serán extraídas del Banco Mundial. Cabe mencionar que estas variables también serán transformadas en su versión logarítmica según la especificación del modelo a estimar. Por otro lado, se incluye el área per cápita, el cual será calculado a partir de la información extraída del Banco Mundial.

Finalmente, para la estimación de la ecuación de gravedad, cuyo valor de 

SOBRE LOS NIVELES DE CONTAMINACIÓN

\section{Tabla 2. Tratado de Libre Comercio de los países que contienen cláusulas con reglamentación medioambiental}

\begin{tabular}{|c|c|c|c|c|c|}
\hline $\begin{array}{l}\text { Nombre del Acuerdo } \\
\text { Comercial Regional }\end{array}$ & $\begin{array}{l}\text { Entrada en } \\
\text { vigor }\end{array}$ & $\begin{array}{c}\text { Posee } \\
\text { cláusula } \\
\text { ambiental }\end{array}$ & $\begin{array}{l}\text { Nombre del Acuerdo } \\
\text { Comercial Regional }\end{array}$ & $\begin{array}{c}\text { Entrada en } \\
\text { vigor }\end{array}$ & $\begin{array}{c}\text { Posee } \\
\text { cláusula } \\
\text { ambiental }\end{array}$ \\
\hline $\begin{array}{c}\text { Comunidad del Caribe } \\
\text { (Costa Rica, El Salvador, } \\
\text { Guatemala, Honduras y } \\
\text { Nicaragua) }\end{array}$ & $1 / 08 / 1973$ & 0 & Panamá-Costa Rica & $23 / 11 / 2008$ & 0 \\
\hline $\begin{array}{c}\text { Comunidad Andina } \\
\text { (Bolivia, Ecuador, Colombia } \\
\text { y Perú) }\end{array}$ & $25 / 05 / 1988$ & 0 & Panamá-Honduras & $9 / 01 / 2009$ & 0 \\
\hline $\begin{array}{c}\text { MERCOSUR } \\
\text { (Argentina, Chile, Paraguay } \\
\text { y Uruguay) }\end{array}$ & $17 / 02 / 1991$ & 1 & Estados Unidos-Perú & $1 / 02 / 2009$ & 1 \\
\hline Colombia-México & $13 / 06 / 1994$ & 1 & Chile-Perú & $1 / 03 / 2009$ & 1 \\
\hline México-Costa Rica & $1 / 01 / 1995$ & 0 & Chile-Australia & $6 / 03 / 2009$ & 1 \\
\hline MERCOSUR-Bolivia & $1 / 01 / 1996$ & 0 & Chile-Colombia & $8 / 05 / 2009$ & 1 \\
\hline MERCOSUR-Chile & $1 / 01 / 1996$ & 0 & Panamá-Guatemala & $20 / 06 / 2009$ & 1 \\
\hline Canadá-Chile & $5 / 07 / 1997$ & 1 & Canadá-Perú & $1 / 08 / 2009$ & 1 \\
\hline México-Nicaragua & $1 / 07 / 1998$ & 0 & $\begin{array}{l}\text { Colombia-Triángulo Norte } \\
\text { (Guatemala, Honduras y El } \\
\text { Salvador) }\end{array}$ & $12 / 11 / 2009$ & 1 \\
\hline México-Chile & $1 / 08 / 1999$ & 1 & Panamá-Nicaragua & $21 / 11 / 2009$ & 1 \\
\hline México-Unión Europea & $1 / 10 / 2000$ & 1 & Perú-China & $1 / 03 / 2010$ & 1 \\
\hline México-Guatemala & $15 / 03 / 2001$ & 0 & Chile-Guatemala & $23 / 03 / 2010$ & 1 \\
\hline México-Honduras & $1 / 06 / 2001$ & 0 & México-Bolivia & $7 / 06 / 2010$ & 0 \\
\hline EFTA-México & $1 / 07 / 2001$ & 0 & Chile-Turquía & $1 / 03 / 2011$ & 1 \\
\hline Chile-Costa Rica & $15 / 02 / 2002$ & 0 & EFTA-Colombia & $1 / 07 / 2011$ & 1 \\
\hline Chile-El Salvador & $1 / 06 / 2002$ & 0 & EFTA-Perú & $1 / 07 / 2011$ & 1 \\
\hline Canadá-Costa Rica & $1 / 11 / 2002$ & 1 & Perú-Corea & $1 / 08 / 2011$ & 1 \\
\hline Chile-Unión Europea & $1 / 02 / 2003$ & 1 & Canadá-Colombia & $15 / 08 / 2011$ & 1 \\
\hline Panamá-El Salvador & $11 / 04 / 2003$ & 0 & Costa Rica-China & $1 / 12 / 2011$ & 1 \\
\hline EFTA-Chile & $1 / 01 / 2004$ & 1 & México-Perú & $1 / 02 / 2012$ & 1 \\
\hline Estados Unidos-Chile & $1 / 01 / 2004$ & 1 & Japón-Perú & $1 / 03 / 2012$ & 1 \\
\hline Chile-Corea & $1 / 04 / 2004$ & 1 & Panamá-Perú & $1 / 05 / 2012$ & 1 \\
\hline México-Japón & $1 / 04 / 2005$ & 1 & Estados Unidos-Colombia & $15 / 05 / 2012$ & 1 \\
\hline Estados Unidos-CAFTA & $1 / 03 / 2006$ & 1 & Chile-Nicaragua & $19 / 10 / 2012$ & 1 \\
\hline Chile-China & $1 / 10 / 2006$ & 1 & Estados Unidos-Panamá & $31 / 10 / 2012$ & 1 \\
\hline Chile-Japón & $3 / 09 / 2007$ & 1 & Perú-Unión Europea & $1 / 03 / 2013$ & 1 \\
\hline Chile-Panamá & $7 / 03 / 2008$ & 1 & Perú-Costa Rica & $1 / 06 / 2013$ & 0 \\
\hline Chile-Honduras & $19 / 07 / 2008$ & 1 & Colombia-Unión Europea & $1 / 08 / 2013$ & 1 \\
\hline CARIFORUM-Unión Europea & $1 / 11 / 2008$ & 1 & Ecuador-Unión Europea & $1 / 01 / 2017$ & 1 \\
\hline
\end{tabular}

Fuente: Banco Mundial, Martínez-Zarzoso (2018). 
de un país a partir de la suma de las exportaciones e importaciones dividido por el PBI del país $i$, y tendrá como variables explicativas adicionales al PBI y población de los países involucrados, a los factores geográficos y culturales del país que relacionan a los países involucrados, cuyos datos serán extraídos del Centro de Investigación y Experiencia en la Economía Mundial (CEPII por sus siglas en inglés), y estarán definidas como variables dicotómicas ${ }^{3}$.

\section{Resultados}

Las estimaciones del modelo principal se presentan en la tabla 3. La primera columna presenta los resultados para el nivel de contaminación del dióxido de carbono ( $\mathrm{CO} 2$ ), la segunda columna presenta los resultados para el nivel de contaminación del óxido de nitrógeno (NOx), la tercera columna presenta los resultados para el nivel de contaminación del dióxido de azufre (SO2) y la cuarta columna presenta los resultados para el nivel de contaminación del material particulado de 2.5 micras (PM 2.5).

Respecto a los resultados de la tabla 3, por cada acuerdo bilateral adicional con cláusula medioambiental, la concentración media de dióxido de carbono ponderada por población en América Latina y el Caribe aumenta en $1.8 \%$. Este resultado es significativo al 1\%. Por otro lado, por cada acuerdo bilateral adicional con cláusula medioambiental, la concentración media de óxido de nitrógeno ponderada por población en América Latina y el Caribe aumenta en 4.7\%. Este coeficiente es significativo al 1\%. Asimismo, por cada acuerdo bilateral adicional con cláusula medioambiental, la concentración media de material particulado de 2.5 micras ponderada por población en América Latina y el Caribe aumenta en $2.5 \%$. Este coeficiente es significativo al $1 \%$. Por el contrario, se observa que por cada acuerdo bilateral adicional con cláusula medioambiental, la concentración media del dióxido de azufre ponderado por población de América Latina y el Caribe generó una disminución significativa en $7.7 \%$ al $1 \%$.

En contraste con el análisis realizado por Baghdadi, Martínez-Zarzoso y Zitouna (2013) y Martínez-Zarzoso (2018), esta investigación se centra en cómo es que las cláusulas están afectando a la región de América Latina y el Caribe, por tal motivo, se encuentran diferencias sobre los resultados obtenidos por estos estudios sobre el efecto negativo de la presencia de una cláusula en

La variable de lengua común tendrá el valor de 1 si es que los países $i$ y $j$ comparten un lenguaje común, mientras que en el caso contrario tomará el valor de 0; la variable contigüidad entre países tendrá el valor 1 si es que los países $i$ y $j$ comparten una frontera común, mientras que en el caso contrario tomará el valor de 0 . La variable relación colonial entre países tendrá el valor de 1 si es que los países $i$ y $j$ compartieron una relación colonial, mientras que en el caso contrario tomará el valor de 0 . La variable relación de colonización por el mismo país tendrá el valor de 1 si es que los países $i$ y $j$ comparten un mismo país colonizador en común, mientras que en el caso contrario tomará el valor de 0. 
los contaminantes analizados para países de diferentes continentes. La región de estudio es bastante heterogénea sobre la presencia de acuerdos bilaterales de este tipo, por lo que se espera también efectos diferenciados dentro de los resultados, sujeto a la dinámica económica propia de cada país. Asimismo, la literatura ha determinado que los acuerdos comerciales pueden ser beneficiosos, pero estos deben ser incluidos específicamente en los acuerdos para tal fin, o sino deberán ir acompañados de cláusulas adicionales, como en el caso del NAFTA (Baghdadi, Martínez-Zarzoso \& Zitouna 2013; Ghosh \& Yamarik 2006).

Tabla 3. Impacto de las cláusulas de reglamentación ambiental sobre indicadores de contaminación

\begin{tabular}{|c|c|c|c|c|}
\hline Variables & $\mathrm{CO} 2$ & NOx & SO2 & PM2.5 \\
\hline \multirow[t]{2}{*}{ LnPoblación_exportador } & $0.675^{* * *}$ & $-0.488^{* * *}$ & $10.89 * * *$ & $-0.436^{* * *}$ \\
\hline & $(0.0387)$ & $(0.117)$ & $(0.0877)$ & $(0.0469)$ \\
\hline \multirow[t]{2}{*}{ LnPIB_pc } & $2.831^{* * *}$ & $-1.871^{* * *}$ & $342.5^{* * *}$ & $-0.430^{* * *}$ \\
\hline & $(0.228)$ & $(0.326)$ & $(3.206)$ & $(0.135)$ \\
\hline \multirow[t]{2}{*}{ LnPIB_pc*LnPIB_pc } & $-0.117^{* * *}$ & $0.124^{* * *}$ & $-18.41^{* * *}$ & $0.0315^{* * *}$ \\
\hline & $(0.0128)$ & $(0.0179)$ & $(0.169)$ & $(0.00709)$ \\
\hline \multirow[t]{2}{*}{ RTA_DM } & $0.0174^{* * *}$ & $0.0457^{* * *}$ & $-0.0803^{* * *}$ & $0.0250^{* * *}$ \\
\hline & $(0.00667)$ & $(0.00893)$ & $(0.0261)$ & $(0.00624)$ \\
\hline \multirow[t]{2}{*}{ RTA_NDM } & $0.0235^{* * *}$ & $0.237^{* * *}$ & - & 0.00508 \\
\hline & $(0.00836)$ & $(0.0265)$ & & $(0.0108)$ \\
\hline \multirow[t]{2}{*}{ Open } & $-0.0956^{* * *}$ & $0.141^{* *}$ & -0.0420 & $-0.0991^{* * *}$ \\
\hline & $(0.0306)$ & $(0.0700)$ & $(0.0715)$ & $(0.0279)$ \\
\hline \multirow[t]{2}{*}{ Constante } & $-17.41^{* * *}$ & $23.90^{* * *}$ & $-1,772^{* * *}$ & $10.83^{* * *}$ \\
\hline & $(1.138)$ & $(2.366)$ & $(15.20)$ & $(0.714)$ \\
\hline Observaciones & 13,336 & 10,115 & 765 & 8,258 \\
\hline R-cuadrado & 0.734 & 0.056 & 0.602 & 0.235 \\
\hline Número de ID & 962 & 961 & 102 & 815 \\
\hline
\end{tabular}

Nota: Donde CO2 hace referencia al contaminante de dióxido de carbono; NOx, al contaminante de óxido de nitrógeno; SO2, al contaminante de dióxido de azufre, y PM2.5 al contaminante de material particulado de 2.5 micras. La estimación se realiza con un modelo de datos panel con efectos fijos. Errores estándar entre paréntesis. ${ }^{* * *} \mathrm{p}<0,01,{ }^{* *} \mathrm{p}<0,05,{ }^{*} \mathrm{p}<0,1$. 
Por otro lado, los resultados de la tabla 4 presentan el análisis de la muestra a partir de 2 submuestras según la estructura de comercio, si esta es sur-norte o sur-sur. En principio, se obtiene que por cada acuerdo bilateral adicional con cláusula medioambiental cuando se está en una estructura de comercio norte-sur, la concentración media de dióxido de carbono ponderada por población aumenta en $1.9 \%$ el valor de este contaminante con un nivel de significancia al 5\%; mientras que, si el comercio es de estructura sur-sur, este aumenta en $1.7 \%$ y este coeficiente es significativo al $10 \%$. Por otro lado, se obtiene que por cada acuerdo bilateral adicional con cláusula medioambiental cuando se está en una estructura de comercio norte-sur, la concentración media de óxido de nitrógeno ponderada por población aumenta significativamente en $6.3 \%$ al $1 \%$; mientras que, si el comercio es de estructura sur-sur, este aumenta $3.2 \%$ a un menor nivel de significancia al 5\%.

Respecto al caso del dióxido de azufre, por cada acuerdo bilateral adicional con cláusula medioambiental cuando se está en una estructura de comercio norte-sur, la concentración media ponderada por población en América Latina y el Caribe aumenta en $6.6 \%$, no obstante, este resultado no es significativo, a diferencia del efecto negativo que genera el tener una cláusula adicional medioambiental sobre este contaminante en un comercio sur-sur, que genera una disminución del 8.3\% significativamente al 5\%. Para el material particulado de 2.5 micras, se obtiene que por cada acuerdo bilateral adicional con cláusula medioambiental cuando se está en una estructura de comercio norte-sur, la concentración media ponderada por población en América Latina y el Caribe aumenta en 3.5\% significativo al 1\%; y para el análisis sur-sur no se encuentra un resultado significativo. Por lo que el análisis por tipo de comercio indicaría que solo la incorporación de disposiciones medioambientales en los acuerdos bilaterales tendrá una incidencia negativa en los sectores relacionados a la emisión de dióxido de azufre y para los países del sur. 


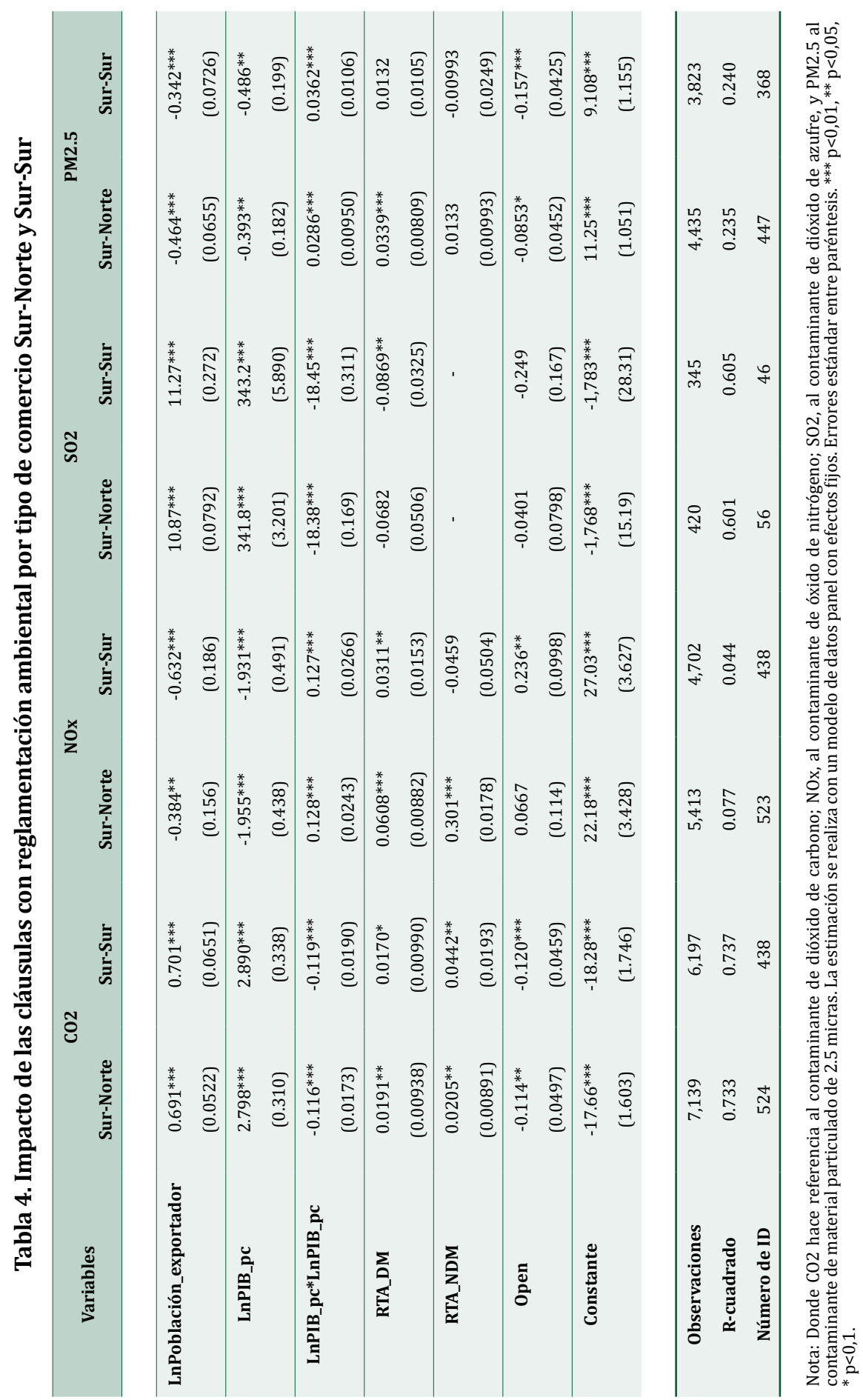


Adicionalmente al análisis realizado, se procede a evaluar cómo es que estas cláusulas ambientales en los acuerdos bilaterales han influido en el comercio de los países que conforman la Alianza del Pacífico, como se reporta en la tabla 5. Los resultados muestran que por cada acuerdo bilateral adicional con cláusula medioambiental, la concentración media de dióxido de carbono ponderada por población en estos países disminuye en 3.7\% y este resultado es significativo al 1\%; a diferencia de cuando se analizaba el caso de América Latina, en el cual el resultado fue significativo y positivo. Por otro lado, si se analiza el efecto sobre el dióxido de azufre, se encuentra que la reducción se genera en una mayor proporción con un valor de 7.7\%, el cual es similar al encontrado también para América Latina. En el caso del dióxido de carbono y material particulado de 2.5 micras, los resultados no fueron significativos.

Tabla 5. Impacto de las cláusulas con reglamentación ambiental para países de la Alianza del Pacífico

\begin{tabular}{ccccc}
\hline Variables & CO2 & NOx & SO2 & PM2.5 \\
\hline LnPoblación_exportador & $0.231^{* * *}$ & $0.685^{* * *}$ & $10.89^{* * *}$ & $-1.788^{* * *}$ \\
& $(0.0224)$ & $(0.0684)$ & $(0.0877)$ & $(0.0540)$ \\
\hline LnPIB_pc & $2.302^{* * *}$ & $-1.120^{* * *}$ & $342.5^{* * *}$ & $-2.020^{* * *}$ \\
& $(0.0767)$ & $(0.119)$ & $(3.206)$ & $(0.143)$ \\
\hline LnPIB_pc*LnPIB_pc & $-0.0702^{* * *}$ & $0.0815^{* * *}$ & $-18.41^{* * *}$ & $0.149^{* * *}$ \\
& $(0.00482)$ & $(0.00712)$ & $(0.169)$ & $(0.00819)$ \\
\hline RTA_DM & $-0.0380^{* * *}$ & 0.00287 & $-0.0803^{* * *}$ & 0.00560 \\
\hline RTA_NDM & $(0.00852)$ & $(0.00488)$ & $(0.0261)$ & $(0.00628)$ \\
\hline Open & 0.00744 & $-0.0257^{* * *}$ & - & $0.0295^{* *}$ \\
& $(0.0163)$ & $(0.00979)$ & & $(0.0132)$ \\
\hline Rúmero de ID & -0.00331 & -0.0306 & -0.0420 & -0.00251 \\
\hline Constante & $(0.0130)$ & $(0.0288)$ & $(0.0715)$ & $(0.0335)$ \\
\hline & $-7.671^{* * *}$ & 0.984 & $-1,772^{* * *}$ & $40.39^{* * *}$ \\
\hline & $(0.614)$ & $(1.426)$ & $(15.20)$ & $(1.352)$ \\
\hline & 204 & & & 2,192 \\
\hline & 3,006 & 2,241 & 765 & 0.658 \\
\hline & 0.879 & 0.754 & 0.602 & 204 \\
\hline
\end{tabular}

Nota: Donde CO2 hace referencia al contaminante de dióxido de carbono; NOx, al contaminante de óxido de nitrógeno; SO2, al contaminante de dióxido de azufre, y PM2.5 al contaminante de material particulado de 2.5 micras. La estimación se realiza con un modelo de datos panel con efectos fijos. Errores estándar entre paréntesis. ${ }^{* * *} \mathrm{p}<0,01,{ }^{* *} \mathrm{p}<0,05,{ }^{*} \mathrm{p}<0,1$. 
Si se hace la diferenciación en la estimación previa para los países socios de la Alianza del Pacífico, diferenciando por el tipo de comercio, nortesur y sur-sur, los resultados varían por esta desagregación, y se muestran en la tabla 6. Para el caso del dióxido de carbono, se obtiene que por cada acuerdo bilateral adicional con cláusula medio ambiental cuando se está en una estructura de comercio sur-norte, la concentración media ponderada por población para estos países reduce $6.2 \%$ y este resultado es significativo al $1 \%$. Respecto al caso del dióxido de azufre, por cada acuerdo bilateral adicional con cláusula medio ambiental cuando se está en una estructura de comercio sur-sur, el valor del contaminante disminuye en $8.3 \%$ significativamente al $5 \%$. Para el caso de los otros dos contaminantes analizados, no se encuentra un efecto significativo de las cláusulas.

Cuando se analizan los resultados obtenidos para la Alianza del Pacífico, se encuentra que, en particular para este grupo de países que ya dispone de acuerdo bilaterales con países desarrollados como los Estados Unidos o la Unión Europea (entre otros), la presencia de estas cláusulas no solo permite reducir la contaminación que es generada por el dióxido de carbono y dióxido de azufre, sino que, en particular, se puede evidenciar que la reducción sobre la cantidad de dióxido de carbono está sujeta a un comercio sur-norte, y que la disminución del dióxido de azufre está sujeto a un comercio sur-sur. No obstante, como se mencionó previamente, el efecto negativo estará sujeto también al detalle sobre las propias cláusulas, que puede variar entre los países, y no fue posible aproximarse de forma más específica a esta variable de acuerdos con la desagregación que se requiere. Por tal motivo, los resultados no resultan concluyentes para todos los casos analizados y esta es una limitación también mencionada dentro del estudio de Martínez-Zarzoso (2018). 
ReVista KaWSAyPaCHa. Sociedad y MedioAmbiente Nº 8 (2021)

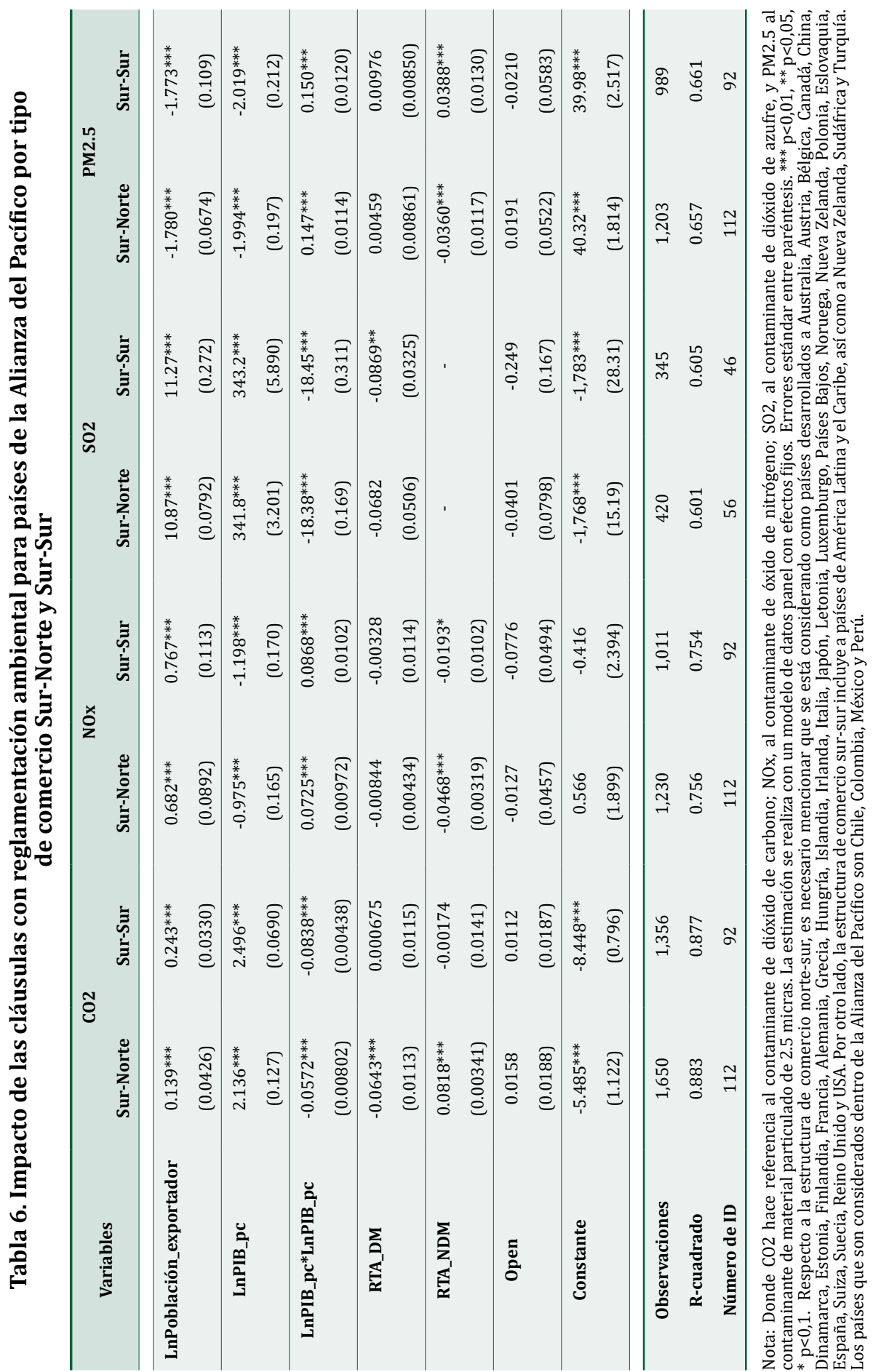




\section{Balance}

El avance del cambio climático, y la vulnerabilidad de la región ante sus efectos, hace necesario que se establezcan cada vez más medidas de adaptación y mitigación, además de promover políticas ambientales que lo contrarresten.

Los resultados de nuestra estimación muestran que, por cada acuerdo bilateral adicional con cláusula medioambiental suscrito en la región, la concentración media de dióxido de carbono ponderada por población aumenta en $1.8 \%$, la concentración media de óxido de nitrógeno aumenta en $4.7 \%$ y la concentración media de material particulado de 2.5 micras aumenta en $2.5 \%$. Sin embargo, se encuentra una disminución del 7.7\% de dióxido de azufre. Si bien el análisis realizado por Baghdadi, Martínez-Zarzoso y Zitouna (2013) y Martínez-Zarzoso (2018) encuentra en todas estas variables una relación indirecta entre los TLC con cláusulas ambientales y los contaminantes analizados, nuestros resultados están asociados a la heterogeneidad que existe en la región sobre la presencia de acuerdos bilaterales de este tipo, por lo que se espera también efectos diferenciados dentro de los resultados sujetos a la dinámica económica propia de cada país.

En el caso de una estructura comercial sur-sur, se genera una disminución del 8.3\%. Por tanto, se puede señalar que la incorporación de disposiciones medioambientales en los acuerdos bilaterales solo tendrá una incidencia negativa en los sectores relacionados a la emisión de dióxido de azufre y para los países del sur.

Asimismo, para la Alianza del Pacífico los resultados muestran que, por cada acuerdo bilateral adicional con cláusula medioambiental, la concentración media ponderada por población de dióxido de carbono disminuye en 3.7\%, al igual que el dióxido de azufre, que se reduce en $7.7 \%$. Este efecto parece sustentarse en que los países que conforman este bloque económico ya disponen de acuerdos bilaterales con países desarrollados, como los Estados Unidos y la Unión Europea, en donde se establecen disposiciones ambientales para reducir la emisión de estos contaminantes.

Sin embargo, es necesario garantizar el cumplimiento efectivo de estas cláusulas para que el impacto sea aún mayor, sobre todo en el actual contexto donde los países de la región deben impulsar un desarrollo sostenible, más verde y resiliente, buscando un equilibrio entre el desarrollo económico y el medio ambiente. 


\section{Referencias}

Banco Mundial (2021). Indicadores de desarrollo mundial. Banco Mundial [Web]. Fecha de consulta: 30 de enero del 2021. http://databank.bancomundial. org/data/home.aspx

Baghdadi, L.; Martínez-Zarzoso, I.; \& Zitouna, H. (2013). Are RTA agreements with environmental provisions reducing emissions? Journal of International Economics, 90(2), 378-390.

Baumann, R. (2016). Acuerdos hechos a medida. El camino a la flexibilidad. Revista Integración \& Comercio, (40).

Grazzi, M. \& Sasso, S. (29 de julio de 2020). Economía circular e innovación verde, pilares para la recuperación y la resiliencia post COVID-19. Puntos sobre la i: Un blog del BID para conversar sobre innovación en América Latina [Blog]. División de Competitividad, Tecnología e Innovación del BID. https://blogs. iadb.org/innovacion/es/economia-circular-innovacion-verde-post-covid-19/

CEPAL (2020). La emergencia del cambio climático en América Latina y el Caribe: ¿seguimos esperando la catástrofe o pasamos a la acción? Santiago de Chile.

CooperAcción (28 de mayo de 2020). Nueva transparencia para nuevos tiempos. Información económica, social y ambiental de las industrias extractivas a la que es indispensable acceder en tiempos de COVID-19. CooperAcción [Web]. https://cooperaccion.org.pe/nueva-transparencia-para-nuevos-tiemposinformacion-economica-social-y-ambiental-de-las-industrias-extractivas-a-laque-es-indispensable-acceder-en-tiempos-de-covid-19/

Ergon Associates (2011). Trade And Labour: Making effective use of trade sustainability impact assessments and monitoring mechanisms. Final Report to DG Employment, Social Affairs and Inclusion. London: Ergon Associates Limited.

Ghosh, S. \& Yamarik, S. (2006). Do regional trading arrangements harm the environment? An analysis of 162 countries in 1990. Applied Econometrics and International Development, 6(2), 15-36.

Hoffmann, B. (2020) Cambio climático y desastres naturales: Exposición desigual, impactos y capacidad para hacerles frente. En M. Busso, \& J. Messina (Eds.). La crisis de la desigualdad: América Latina y el Caribe en la encrucijada (pp. 248268). Banco Interamericano de Desarrollo.

Hoffmann, B. (30 de abril de 2021). Cómo el cambio climático empeora la pobreza y la desigualdad. Ideas que cuentan [Blog]. Banco Interamericano de Desarrollo. https://blogs.iadb.org/ideas-que-cuentan/es/como-el-cambio-climaticoempeora-la-pobreza-y-la-desigualdad/

Manero, A. (2020). La política comercial común de la Unión Europea y el desarrollo sostenible. Revista de Derecho Comunitario Europeo, 66, 603-627.

Martínez-Zarzoso, I. (2017). El impacto ambiental de los tratados comerciales. EcoIntegración de América Latina, 162-176.

Martínez-Zarzoso, I. (2018). Assessing the effectiveness of environmental provisions in regional trade agreements: An empirical analysis. OECD Library. 
Mattoo, A.; Rocha, N.; \& Ruta, M. (Eds). (2020). Manual de acuerdos comerciales profundos. Washington: Banco Mundial. https://openknowledge.worldbank. org/handle/10986/34055

Naciones Unidas (2015). Objetivo 17: Revitalizar la Alianza Mundial para el Desarrollo Sostenible. https://www.un.org/sustainabledevelopment/es/globalpartner ships/

Naciones Unidas (2020). World Economic Situation Prospects. https://www.un.org/ development/desa/d pad/wp-content/uploads/sites / 45 /WESP2020_Annex.pdf

OECD (2021). Air and GHG emissions. Recuperado de OECD Data. Fecha de Consulta: 30 de enero del 2021. Enlace: https://data.oecd.org/air/air-and-ghg-emissions. htm\#indicator-chart

OMC (10 de julio de 2021a). Comercio y medio ambiente. Sección Temas Comerciales, Organización Mundial de Comercio [Web]. https://www.wto.org/spanish/ tratop_s/envir_s/envir_s.htm

OMC (10 de julio de 2021b). Introducción al comercio y el medio ambiente en la OMC. Sección Temas Comerciales, Organización Mundial de Comercio [Web]. https:// www.wto.org/spanish/tratop_s/envir_s/envt_intro_s.htm

OMC (2020). Short Answers To Big Questions: On the WTO and environment. Organización Mundial del Comercio.

Salazar,C. (18 de junio de 2020). ¿Es posible incluir un capítulo ambiental en el TLC Perú-China? Comunidad Informativa [Web]. Derecho, Ambiente y Recursos Naturales (DAR). https://dar.org.pe/es-posible-incluir-un-capitulo-ambientalen-el-tlc-peru-china/

UNCTAD (2021a) Trade Statistics. Recuperado de UN Comtrade Database. Fecha de consulta: 30 de enero de 2021. Enlace: https://comtrade.un.org/data/

UNCTAD (2021b). Trade and Environment Review 2021. United Nations Publications. 